\title{
Association between insulin and executive functioning in alcohol dependence: a pilot study
}

This article was published in the following Dove Press journal:

Neuropsychiatric Disease and Treatment

16 November 2015

Number of times this article has been viewed

\author{
Changwoo Han' \\ Hwallip Bae ${ }^{2}$ \\ Sung-Doo Won ${ }^{3}$ \\ Jaeyoung $\mathrm{Lim}^{3}$ \\ Dai-Jin $\mathrm{Kim}^{4}$ \\ 'Department of Psychiatry, Ansan \\ Hospital, College of Medicine, Korea \\ University, Ansan, ${ }^{2}$ Department \\ of Psychiatry, Myongji Hospital, \\ College of Medicine, Seonam \\ University, Goyang, ${ }^{3}$ Department of \\ Clinical Psychology, Keyo Hospital, \\ Keyo Medical Foundation, Uiwang, \\ ${ }^{4}$ Department of Psychiatry, College of \\ Medicine, The Catholic University of \\ Korea, Seoul, Republic of Korea
}

Correspondence: Dai-Jin Kim Department of Psychiatry, Seoul St Mary's Hospital, College of Medicine, The Catholic University of Korea, 222 Banpo-daero, Seocho-gu,

Seoul I37-70I, Republic of Korea

Tel +82222586086

Fax +82 25943870

Email kdj922@catholic.ac.kr
Abstract: Alcohol dependence is a disorder ascribable to multiple factors and leads to cognitive impairment. Given that insulin dysregulation can cause cognitive impairment, patients with alcohol dependence are likely to develop insulin dysregulation such as that in diabetes. The purposes of this study are to identify an association between cognitive functioning and insulin and to investigate insulin as the biomarker of cognitive functioning in alcohol-dependent patients. Serum insulin levels were measured and cognitive functions were assessed in 45 patients with chronic alcoholism. The Korean version of the Consortium to Establish a Registry for Alzheimer's Disease (CERAD-K), a battery of cognitive function tests, was used to assess cognitive functioning. Serum insulin levels were not significantly correlated with most CERAD-K scores, but there was a significant negative correlation with scores on the Trail Making Test B, which is designed to measure executive functioning. Lower serum insulin levels were associated with slower executive functioning responses on the Trail Making Test B, suggesting that executive functioning may be in proportion to serum insulin levels. Thus, in patients with alcohol dependence, insulin level is associated with cognitive functioning. In addition, the present findings suggest that insulin level is a potential biomarker for determining cognitive functioning.

Keywords: insulin, alcohol dependence, executive function, trail making test

\section{Introduction}

Insulin, a major metabolic hormone, is secreted by the beta cells in the pancreatic islets of Langerhans and maintains constant blood glucose levels. In peripheral tissues, insulin regulates glucose uptake, oxidation, and storage. Additionally, insulin has been established as a contributor to cognitive functioning. Cognitive impairment occurs in diseases associated with underlying insulin dysfunction, including diabetes, hyperlipidemia, hypertension, and obesity. ${ }^{1}$ Insulin receptors are widely distributed in several organs, with high concentrations in the limbic system. ${ }^{2}$ The limbic system is involved in motivation, emotion, learning, and memory. In the brain, insulin receptors are mainly observed at the synapses of neurons and astrocytes. Insulin signaling involves synaptogenesis and synaptic remodeling. ${ }^{3}$

Insulin is directly involved in neurotransmitter expression (eg, acetylcholine or norepinephrine), which explains its involvement in cognitive functioning. ${ }^{4,5}$ Further, insulin modulates glucose metabolism in other organs and increases cerebral glucose metabolism, which may be a potential mechanism for its involvement in cognition. ${ }^{6}$

Alcohol use disorder results in substantial damage to the central nervous system in many patients. ${ }^{7}$ Long-term alcohol use contributes to several somatic symptoms, including hepatic dysfunction or endocrine disorders, ${ }^{8}$ and excessive alcohol intake contributes to acute or chronic pancreatitis. 
In addition, chronic and excessive alcohol consumption decreases glucose tolerance, which increases the risk of developing type II diabetes. ${ }^{9}$ Patients with alcohol dependence who have comorbid diabetes performed poorly on language and symbol tests compared to nondiabetic patients with alcohol dependence. ${ }^{10}$

Significant decline in cognitive functioning is known to occur in patients with alcohol dependence. Simple learning or memory functions can recover over time, whereas abstract reasoning, problem-solving skills, and perceptual-motor skills remained lower than nonalcohol-dependent controls even after abstaining from alcohol for 1 year. ${ }^{11}$

Based on previous studies, neurocognitive impairment in alcohol-dependent patients could have the relationship with insulin and glucose intolerance. Therefore, it is possible to suggest that the effect of insulin on the glucose metabolism is a crucial reason of the cognitive decline in alcohol dependence. Although details of the relationship between alcohol dependence and insulin have not been fully elucidated, ${ }^{12}$ when compared to normal controls, patients with alcohol dependence had higher levels of insulin and C-peptides. ${ }^{13}$ Another study of patients with alcohol dependence established a contribution of insulin and found a significant correlation between scores on an Obsessive-Compulsive Drinking Scale and C-peptide level. ${ }^{14}$

Nevertheless, insulin would have a major role in neurocognitive function in alcohol dependence, and there were less studies about the insulin and the cognitive functioning of alcohol-dependent patients. This study tried to identify the relationship between insulin and cognitive functioning in nondiabetic alcohol-dependent patients. It is noteworthy that insulin could be a potential indicator that reflects the cognitive impairment in alcohol-dependent patients.

Despite the findings described earlier, few studies have investigated the relationship between insulin and cognitive functioning in patients with alcohol dependence. Insulin like growth factor-1 (IGF-1), which functionally parallels insulin, has significant effects on cognitive functioning in patients with alcohol dependence. ${ }^{15}$ IGF-1 is a polypeptide that is structurally related to insulin and has an increased activity in the presence of growth hormone. ${ }^{16}$ Given that the activity of IGF-1 is similar to that of insulin, a relationship between insulin and alcohol dependence is likely and may explain deteriorating cognitive functioning in patients with alcohol dependence.

This study aimed to identify a correlation between insulin level and cognitive functioning in patients with alcohol dependence by measuring insulin levels and assessing cognitive functioning. Accordingly, this study attempts to determine the potential of insulin for use as the biological marker of cognitive decline in alcohol-dependent patients.

\section{Patients and methods Subjects}

This study was conducted among 45 patients hospitalized for alcohol rehabilitation treatment in a psychiatric hospital located in the Gyeonggi Province of South Korea. Participants were diagnosed with alcohol dependence by two psychiatrists, based on DSM-IV-TR criteria. Participants with current or historical diabetes were excluded. Participants whose blood glucose levels were $200 \mathrm{mg} / \mathrm{dL}$ or higher and those who had neurological disorders including a history of severe head injuries were also excluded. Only patients who were confirmed as abstinent from alcohol for at least 1 week were included. Patients with current alcohol withdrawal symptoms were excluded.

The present study was approved by the Institutional Research Board of Seoul St Mary's Hospital (C11QISI0569). All participants were men, and they voluntarily signed informed consent after being informed of the study purpose.

\section{Clinical assessment}

Participants were surveyed in order to collect data on demographic and clinical variables, including age, education, onset age of drinking, and Korean version of Alcohol Dependence Scale (ADS-K). ${ }^{17}$ ADS-K is a scale consisting of a total of 25 items developed by Skinner and Allen through factor analysis. The scale was designed to assess compulsive drinking, behavior control when drinking, and alcohol withdrawal symptoms.

Cognitive functioning was assessed with the Korean Version of the Consortium to Establish a Registry for Alzheimer's Disease (CERAD-K) battery of neuropsychological tests. ${ }^{18}$ These neuropsychological tests assess language, memory, and construction and performance skills, and it is composed of nine items (verbal fluency, word list memory, word list recall, word list recognition, constructional praxis, constructional recall, Boston Naming Test, Korean Version of Mini-Mental State Examination, and trail making test). ${ }^{19}$ In the present study, two clinical laboratory technologists tested each patient.

\section{Enzyme-linked immunosorbent assay}

An EDTA-coated tube treated with $10 \mu \mathrm{L} / \mathrm{mL}$ of Pefabloc SC (Roche Diagnostics GmbH, Mannheim, Germany) was used to collect $10 \mathrm{~mL}$ of fasting blood. This sample was used for baseline analyses of several factors. The blood was 
centrifuged immediately at $2,500 \times g$ for 10 minutes. Then, the plasma samples were stored at $-80^{\circ} \mathrm{C}$ until assayed.

Liver enzymes in plasma, including aspartate aminotransferase, alanine aminotransferase, and alkaline phosphatase, were assayed using an enzyme activity assay (Daiichi Sankyo Company, Tokyo, Japan). High-density lipoprotein and low-density lipoprotein cholesterol levels were measured by selective inhibition methods (Daiichi Sankyo Company) and triglycerides were measured using a glycerol blanking method (Wako Pure Chemical Industries, Osaka, Japan). Insulin level was measured using enhanced chemiluminescence (Roche Diagnostics Korea, Seoul, South Korea), and glucose was assayed by a hexokinase UV method (Asan Pharmaceutical, Seoul, South Korea). All assays were performed according to manufacturers' instructions, using the recommended buffers, diluents, and substrates. The sample concentration in each plate was calculated according to appropriate standard curves and dilution factors.

\section{Statistical analyses}

Descriptive statistics were used to analyze demographic characteristics. Correlation analyses were performed in order to determine the correlations between CERAD-K cognitive test results and blood assay values.

Statistical analyses were performed using SPSS (Statistical Package for Social Sciences) version 16.0. A significance level of $P<0.05$ was applied in all analyses.

\section{Results}

\section{Demographic variables and alcohol dependence}

Table 1 presents the demographic and clinical characteristics of participants. Participants were 38-61 years old. The mean age of participants was 51 years, and they had an average education level of 10 years. The mean onset age of drinking was 22 years and the mean ADS-K score was 22, which is higher than expected in a nondependent population.

\section{Relationship between alcohol dependence and neurocognitive functioning}

The mean of Korean Version of Mini-Mental State Examination scores of patients with alcohol dependence was 26.76 . The mean interference index, which indicates declining executive functioning, was 2.78. This score indicates that participants took three times longer to perform the Trail Making Test B than they took to perform the Trail Making Test A (Table 2).
Table I Demographic and clinical characteristics of patients with alcohol dependence

\begin{tabular}{ll}
\hline & Mean \pm SD \\
\hline Age (years) & $51.20 \pm 6.26$ \\
Education (years) & $10.00 \pm 3.64$ \\
Onset age of drinking (years) & $22.35 \pm 8.51$ \\
ADS-K & $22.22 \pm 7.22$ \\
AST (IU/L) & $23.00 \pm 7.89$ \\
ALT (IU/L) & $23.96 \pm 15.73$ \\
ALP (IU/L) & $72.02 \pm 19.93$ \\
Triglyceride (mg/dL) & $190.51 \pm 88.92$ \\
HDL-cholesterol (mg/dL) & $43.78 \pm 11.66$ \\
LDL-cholesterol (mg/dL) & $104.84 \pm 25.06$ \\
Glucose $(\mathrm{mg} / \mathrm{dL})$ & $125.98 \pm 53.56$ \\
Insulin $(\mu / \mathrm{m} / \mathrm{mL})$ & $31.67 \pm 31.22$ \\
\hline
\end{tabular}

Abbreviations: ADS-K, Korean version of the Alcohol Dependence Scale; SD, standard deviation; AST, aspartate aminotransferase; ALT, alanine aminotransferase; ALP, alkaline phosphatase; HDL-cholesterol, high-density lipoprotein cholesterol; LDL-cholesterol, low-density lipoprotein cholesterol.

\section{Relationship between insulin level and neurocognitive functioning in patients with alcohol dependence}

Insulin level and ADS-K score were not significantly correlated. In addition, there were no significant correlations between insulin level and most CERAD-K subtest scores. However, scores for the Trail Making Test B, which indicates executive functioning levels, was significantly correlated with insulin levels ( $r=-0.329, P<0.05)$, with lower subtest scores associated with higher insulin levels (Table 3 ).

\section{Discussion}

The present study aimed to identify correlations between cognitive functioning and insulin levels in patients with alcohol dependence. The participants had relatively stable

Table 2 Results of CERAD-K and correlations between insulin level and CERAD-K score

\begin{tabular}{ll}
\hline CERAD-K & Mean \pm SD \\
\hline Verbal fluency & $15.11 \pm 3.62$ \\
Word list memory & $18.18 \pm 4.07$ \\
Word list recall & $6.39 \pm 2.09$ \\
Word list recognition & $9.34 \pm 0.94$ \\
Constructional praxis & $10.42 \pm 1.18$ \\
Constructional recall & $8.76 \pm 2.54$ \\
Boston Naming Test & $12.10 \pm 1.94$ \\
MMSE-K & $26.76 \pm 3.09$ \\
TMT A & $51.42 \pm 24.78$ \\
TMT B & $194.47 \pm 92.84$ \\
Interference index of TMT & $2.78 \pm 1.65$ \\
\hline
\end{tabular}

Abbreviations: CERAD-K, Korean version of the Consortium to Establish a Registry for Alzheimer's Disease; MMSE-K, Korean Version of Mini-Mental State Examination; TMT, trail making test; SD, standard deviation. 
Table 3 Correlations between insulin level and CERAD-K scores

\begin{tabular}{lll}
\hline CERAD-K & $\begin{array}{l}\text { Pearson correlation } \\
\text { with insulin level }\end{array}$ & P-value \\
\hline Verbal fluency & 0.155 & 0.354 \\
Word list memory & 0.127 & 0.446 \\
Word list recall & 0.246 & 0.137 \\
Word list recognition & 0.138 & 0.407 \\
Constructional praxis & 0.004 & 0.983 \\
Constructional recall & -0.068 & 0.687 \\
Boston Naming Test & -0.158 & 0.343 \\
MMSE-K & 0.131 & 0.433 \\
TMT A & -0.264 & 0.109 \\
TMT B & $-0.329 *$ & 0.044 \\
\hline
\end{tabular}

Note: $* P<0.05$.

Abbreviations: CERAD-K, Korean version of the Consortium to Establish a Registry for Alzheimer's Disease; MMSE-K, Korean Version of Mini-Mental State Examination; TMT, trail making test.

scores on most subtests of the CERAD-K, and there were no correlations between insulin level and most CERAD-K subtest scores. However, scores on the Trail Making Test B, which reflect the speed of executive functioning, were associated with higher insulin levels. There were no relationships between demographic variables and cognitive functioning. A previous study reported that patients with problematic alcohol use and posttraumatic stress disorder showed the association between elevated levels of aspartate aminotransferase and trail making A test. ${ }^{20}$ But in the present study, clinical datum did not show any relationship with cognitive functioning.

Trail making tests involve motor components and complex visual scanning and can be used to assess visual-motor tracking. These tests require sustained attention and psychomotor speed. ${ }^{21,22}$ The Trail Making Test B requires more complex mental processes compared to the Trail Making Test $\mathrm{A}$ and is known to be more sensitive to symptoms of brain injury. ${ }^{23,24}$ In the present study, insulin was correlated with the Trail Making Test B, not the Trail Making Test A. The result suggests that insulin would reflect rather complex executive functioning than simple cognition. That is, lower serum insulin levels were associated with slower executive functioning responses on the Trail Making Test B. In the previous study that alcohol-dependent patients who have comorbid diabetes showed lower cognitive functioning compared to nondiabetic patients with alcohol dependence. ${ }^{10} \mathrm{By}$ the result of the present study, in case of nondiabetic alcoholdependent patients, insulin also could reflex the cognitive decline in alcohol dependence. Therefore, insulin related to glucose metabolism would be one of the important mechanism the excessive alcohol drinking induce neurodegenesis in alcohol dependence.
Participants did not have higher education, and there was no significant difference in education levels, with a standard deviation of 3.6 years despite the mean of 10 years. Regarding the interference index between trail making tests, it took three times longer to complete the Trail Making Test B compared to the trail making A test, which indicates declining mental set-shifting abilities in patients with alcohol dependence.

Deterioration in cognitive functioning in these patients is attributable to multiple factors, including cranial nerve injuries ascribable to alcohol. Chronic alcohol intake is known to cause both temporary cognitive impairment and permanent structural damage. ${ }^{25}$ Previous research has found that $45 \%$ of patients with alcohol dependence had cognitive impairment even after several weeks of abstinence from alcohol, and $15 \%$ of these patients had impairment even after abstinence for 1 year. ${ }^{26}$ In particular, declines are observed in cognitive functions related to learning and memory. ${ }^{27}$ Psycho-motor deficits, circumstantiality, attention deficits, and orientation disorders have been observed. ${ }^{28}$ In particular, chronic alcohol use may contribute to deteriorating executive functioning related to attention, problem-solving skills, and self-control. ${ }^{29}$ Executive function may be a primary predictor of poor prognosis because patients' social adjustment is decreased and their functional rehabilitation is impaired. ${ }^{30}$

Insulin may improve cognitive functioning, including memory. In particular, patients with Alzheimer's disease often experience insulin resistance and severe neurodegeneration in conjunction with declining insulin level. ${ }^{31}$ However, when brain insulin levels increase, cognitive functions improve. ${ }^{32}$ One study demonstrated treatment effects on cognitive functioning by administering insulin to patients with diseases that included cognitive impairment. ${ }^{33}$ Another study compared changes in cognitive functioning following insulin injection, using a trail making test. ${ }^{34}$ In this study, intranasal administration of insulin to patients with bipolar disorder, followed by a trail making test, indicated a significant improvement in executive functioning compared to placebo groups.

Cognitive impairment has been evaluated in patients with concurrent diabetes and alcohol dependence. ${ }^{10}$ In this study, patients took an oral glucose tolerance test and were then categorized into diabetic and nondiabetic groups prior to completing the CERAD-K and K-WAIS (Korean Wechsler Adult Intelligence Scale) tests. Diabetic patients with alcohol dependence had lower executive functioning and language scores. 
Further, in patients with alcohol dependence whose hepatic cells were histologically normal, indicating that they had developed no diabetic complications, insulin levels were higher compared to normal controls. ${ }^{13}$ This finding suggests that patients with alcohol dependence were highly likely to develop impaired glucose tolerance and beta cell dysfunction. Therefore, alcohol dependence and insulin abnormalities can be associated with cognitive impairment.

\section{Conclusion}

The present study verified that insulin levels of patients with alcohol dependence were relevant to performance on the Trail Making Test B, which indicates executive cognitive functioning. Therefore, patients with alcohol dependence, even without comorbid diabetes, may have impaired glucose tolerance and be at risk of developing cognitive impairments. Insulin may therefore be a useful biomarker for impaired cognitive functioning.

\section{Limitations}

The present study has limitations. First, this study used the CERAD to measure cognitive functioning. Further studies are needed to adapt tools to help assess more complex cognitive abilities among alcohol-dependent patients. The present small sample composed entirely of men is another limitation. And this study was a lack of comparison between alcohol-dependent patients and the control group. Because excessive alcohol consumption is associated with a range of medical manifestations, comparing the datum of normal control to those of alcohol-dependent patients is needed to make the result more clear. Finally, the longitudinal design is needed. Although our study tried to exclude the influence of alcohol withdrawal symptoms, the cognitive functions of the alcohol-dependent patients were changeable during their admission. These limitations warrant further investigation of the relationship between insulin level and executive functioning in patients with alcohol dependence.

\section{Acknowledgments}

The study was supported by a grant from the Korean Health Technology R\&D Project, Ministry of Health and Welfare, Republic of Korea (HI11C1332). The authors wish to acknowledge the financial support of the Catholic Medical Center Research Foundation made in the program year of 2011.

\section{Disclosure}

The authors report no conflicts of interest in this work.

\section{References}

1. Strachan MW. Insulin and cognitive function. Lancet. 2003;362(9392): 1253.

2. Freychet $P$. Insulin receptors and insulin actions in the nervous system. Diabetes Metab Res Rev. 2000;16(6):390-392.

3. Abbott M-A, Wells DG, Fallon JR. The insulin receptor tyrosine kinase substrate $\mathrm{p} 58 / 53$ and the insulin receptor are components of CNS synapses. J Neurosci. 1999;19(17):7300-7308.

4. Figlewicz DP, Szot P, Israel PA, Payne C, Dorsa DM. Insulin reduces norepinephrine transporter mRNA in vivo in rat locus coeruleus. Brain Res. 1993;602(1):161-164.

5. Kopf SR, Baratti CM. Effects of posttraining administration of insulin on retention of a habituation response in mice: participation of a central cholinergic mechanism. Neurobiol Learn Mem. 1999;71(1):50-61.

6. Bingham EM, Hopkins D, Smith D, et al. The role of insulin in human brain glucose metabolism an 18 fluoro-deoxyglucose positron emission tomography study. Diabetes. 2002;51(12):3384-3390.

7. Harper C, Matsumoto I. Ethanol and brain damage. Curr Opin Pharmacol. 2005;5(1):73-78.

8. Rehm J. The risks associated with alcohol use and alcoholism. Alcohol Res Health. 2011;34(2):135-143.

9. Wannamethee S, Shaper A, Perry I, Alberti K. Alcohol consumption and the incidence of type II diabetes. J Epidemiol Community Health. 2002;56(7):542-548.

10. Hwang H-R, Min J-A, Kwon M, et al. Neurocognitive function impairment in alcohol dependent patients with diabetes mellitus. $J$ Korean Neuropsychiatr Assoc. 2012;51(5):285-290.

11. Fein G, Bachman L, Fisher S, Davenport L. Cognitive impairments in abstinent alcoholics. West J Med. 1990;152(5):531.

12. Baliunas DO, Taylor BJ, Irving H, et al. Alcohol as a risk factor for type 2 diabetes: a systematic review and meta-analysis. Diabetes Care. 2009;32(11):2123-2132.

13. Andersen BN, Hagen C, Faber O, Lindholm J, Boisen P, Worning H. Glucose tolerance and B cell function in chronic alcoholism: its relation to hepatic histology and exocrine pancreatic function. Metabolism. 1983;32(11):1029-1032.

14. Leggio L, Ferrulli A, Malandrino N, et al. Insulin but not insulin growth factor-1 correlates with craving in currently drinking alcohol-dependent patients. Alcohol Clin Exp Res. 2008;32(3):450-458.

15. Han C, Bae H, Won S-D, Lee HK. Association between insulin-like growth factor-1 and cognitive functions in alcohol-dependent patients. Neurosci Lett. 2014;583:205-209.

16. Tannenbaum GS, Guyda HJ, Posner BI. Insulin-like growth factors: a role in growth hormone negative feedback and body weight regulation via brain. Science. 1983;220(4592):77-79.

17. Lee DK, Shin JK, Byun WT. A reliability and validity study of the Korean version of the alcohol dependence scale in alcoholics. $J$ Korean Acad Addict Psychiatry. 2000;4:30-37.

18. Lee JH, Lee KU, Lee DY, et al. Development of the Korean version of the Consortium to Establish a Registry for Alzheimer's Disease Assessment Packet (CERAD-K): clinical and neuropsychological assessment batteries. The J Gerontol B Psychol Sci Soc Sci. 2002;57(1): P47-P53.

19. Fillenbaum GG, van Belle G, Morris JC, et al. Consortium to Establish a Registry for Alzheimer's Disease (CERAD): the first twenty years. Alzheimers Dement. 2008;4(2):96-109.

20. Kalapatapu RK, Neylan TC, Regan MC, Cohen BE. Association of alcohol use biomarkers and cognitive performance in veterans with problematic alcohol use and posttraumatic stress disorder: data from the mind your heart study. $J$ Addict Dis. 2014;33(2):67-76.

21. Crowe SF. The differential contribution of mental tracking, cognitive flexibility, visual search, and motor speed to performance on parts A and B of the Trail Making Test. J Clin Psychol. 1998;54(5): 585-591.

22. Miner T, Ferraro FR. The role of speed of processing, inhibitory mechanisms, and presentation order in Trail-Making test Performance. Brain Cogn. 1998;38(2):246-253. 
23. Arbuthnott K, Frank J. Trail making test, part B as a measure of executive control: validation using a set-switching paradigm. J Clin Exp Neuropsychol. 2000;22(4):518-528.

24. Gaudino EA, Geisler MW, Squires NK. Construct validity in the Trail Making Test: what makes Part B harder? J Clin Exp Neuropsychol. 1995;17(4):529-535.

25. Fadda F, Rossetti ZL. Chronic ethanol consumption: from neuroadaptation to neurodegeneration. Prog Neurobiol. 1998;56(4):385-431.

26. Rourke, Sean B, Løberg T. Neurobehavioral correlates of alcoholism. In: Grant I, Adams KM, editors. Neuropsychological Assessment of Neuropsychiatric Disorders. New York: Oxford University Press; 1996: 423-485.

27. Parsons OA. Neuropsychological deficits in alcoholics: facts and fancies. Alcohol Clin Exp Res. 1977;1(1):51-56.

28. Lishman W. Cerebral disorder in alcoholism syndromes of impairment. Brain. 1981;104(1):1-20.

29. Zinn S, Stein R, Swartzwelder HS. Executive functioning early in abstinence from alcohol. Alcohol Clin Exp Res. 2004;28(9):1338-1346.
30. Miller L. Predicting relapse and recovery in alcoholism and addiction: neuropsychology, personality, and cognitive style. J Subst Abuse Treat. 1991;8(4):277-291.

31. Rivera EJ, Goldin A, Fulmer N, Tavares R, Wands JR, de la Monte SM. Insulin and insulin-like growth factor expression and function deteriorate with progression of Alzheimer's disease: link to brain reductions in acetylcholine. J Alzheimers Dis. 2005;8(3):247-268.

32. Morris JK, Burns JM. Insulin: an emerging treatment for Alzheimer's disease dementia? Curr Neurol Neurosci Rep. 2012;12(5):520-527.

33. Benedict $\mathrm{C}$, Hallschmid $\mathrm{M}$, Hatke A, etal. Intranasal insulin improves memory in humans. Psychoneuroendocrinology. 2004;29(10):1326-1334.

34. McIntyre RS, Soczynska JK, Woldeyohannes HO, et al. A randomized, double-blind, controlled trial evaluating the effect of intranasal insulin on neurocognitive function in euthymic patients with bipolar disorder. Bipolar Disord. 2012;14(7):697-706.
Neuropsychiatric Disease and Treatment

\section{Publish your work in this journal}

Neuropsychiatric Disease and Treatment is an international, peerreviewed journal of clinical therapeutics and pharmacology focusing on concise rapid reporting of clinical or pre-clinical studies on a range of neuropsychiatric and neurological disorders. This journal is indexed on PubMed Central, the 'PsycINFO' database and CAS,

\section{Dovepress}

and is the official journal of The International Neuropsychiatric Association (INA). The manuscript management system is completely online and includes a very quick and fair peer-review system, which is all easy to use. Visit http://www.dovepress.com/testimonials.php to read real quotes from published authors. 